\title{
PRANDTL-MEYER REFLECTION FOR SUPERSONIC FLOW PAST A SOLID RAMP
}

\author{
MYOUNGJEAN BAE, GUI-QIANG CHEN, AND MIKHAIL FELDMAN \\ Dedicated to Costas Dafermos on the occasion of his 70th birthday
}

\begin{abstract}
We present our recent results on the Prandtl-Meyer reflection for supersonic potential flow past a solid ramp. When a steady supersonic flow passes a solid ramp, there are two possible configurations: the weak shock solution and the strong shock solution. Elling-Liu's theorem (2008) indicates that the steady supersonic weak shock solution can be regarded as a long-time asymptotics of an unsteady flow for a class of physical parameters determined by certain assumptions for potential flow. In this paper we discuss our recent progress in removing these assumptions and establishing the stability theorem for steady supersonic weak shock solutions as the long-time asymptotics of unsteady flows for all the physical parameters for potential flow. We apply new mathematical techniques developed in our recent work to obtain monotonicity properties and uniform apriori estimates for weak solutions, which allow us to employ the LeraySchauder degree argument to complete the theory for the general case.
\end{abstract}

\section{INTRODUCTION}

We are concerned with unsteady global solutions for supersonic flow past a solid ramp, which may be also regarded as portraying the symmetric gas flow impinging onto a solid wedge (by symmetry). When a steady supersonic flow past a solid ramp whose slope is less than a critical slope, Prandtl employed the shock polar analysis to show that there are two possible configurations: the weak shock reflection with supersonic downstream flow and the strong shock reflection with subsonic downstream flow, which both satisfy the entropy conditions, provided that we do not give additional conditions at downstream; see Busemann [3], Courant-Friedrichs [10], Meyer [15], and Prandtl [16].

The fundamental question of whether one or both of the strong and the weak shocks are physically admissible has been vigorously debated over the past seventy years, but has not yet been settled in a definite manner (cf. Courant-Friedrichs [10], Dafermos [11], and Serre [17]). On the basis of experimental and numerical evidence, there are strong indications that it is the Prandtl-Meyer weak reflection

Date: December 31, 2011.

2010 Mathematics Subject Classification. Primary: 35M10, 35M12, 35B65, 35L65, 35L70, 35J70, 76H05, 35L67, 35R35; Secondary: 35L15, 35L20, 35J67, 76N10, 76L05.

Key words and phrases. Prandtl-Meyer reflection, supersonic flow, unsteady flow, steady flow, solid wedge, weak shock solution, strong shock solution, stability, self-similar, transonic shock, sonic boundary, free boundary, existence, regularity, elliptic-hyperbolic mixed, monotonicity, apriori estimates, uniform estimates,... 
solution that is physically admissible. One plausible approach is to single out the strong shock reflection by the consideration of stability, the stable ones are physical. It has been shown in the steady regime that the Prandtl-Meyer weak reflection is not only structurally stable (cf. Chen-Zhang-Zhu [8]), but also $L^{1}$-stable with respect to steady small perturbation of both the ramp slope and the incoming steady upstream flow (cf. Chen-Li [7]), while the strong reflection is also structurally stable for a large spectrum of physical parameters (cf. Chen-Fang [9]). The first rigorous unsteady analysis of the steady supersonic weak shock solution as the long-time behavior of an unsteady flow is due to Elling-Liu in [13] in which they succeeded to establish a stability theorem for a class of physical parameters determined by certain assumptions for potential flow (see §3.1).

The purpose of this work is to remove the assumptions in Elling-Liu's theorem [13] and establish the stability theorem for the steady supersonic weak shock solution as the long-time asymptotics of an unsteady flow for all the admissible physical parameters (without additional conditions) for potential flow. To complete this theorem, we apply new mathematical techniques developed in Chen-Feldman [6] to obtain uniform apriori estimates for weak solutions. We first establish the monotonicity property and its consequence of weak solutions (see $\S 4.1$ ). Then we make various uniform apriori estimates of weak solutions for two cases: the $C^{2, \alpha}$-estimates away from the sonic circles where the governing equation is uniformly elliptic (see §4.3.1)

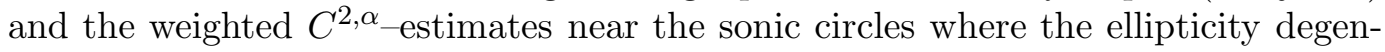
erates (see §4.3.2). These careful estimates allow us to employ the Leray-Schauder degree argument to establish the complete theory (see $\S 4.4$ ).

\section{Mathematical Formulation for the Problem}

The unsteady potential flow is governed by the conservation law of mass and Bernoulli's law:

$$
\begin{aligned}
& \partial_{t} \rho+\nabla_{\mathbf{x}} \cdot\left(\rho \nabla_{\mathbf{x}} \Phi\right)=0, \\
& \partial_{t} \Phi+\frac{1}{2}\left|\nabla_{\mathbf{x}} \Phi\right|^{2}+i(\rho)=B
\end{aligned}
$$

for the density $\rho$ and the velocity potential $\Phi$, where the Bernoulli constant $B$ is determined by the incoming flow and/or boundary conditions, and $i(\rho)$ satisfies the relation

$$
i^{\prime}(\rho)=\frac{p^{\prime}(\rho)}{\rho}=\frac{c^{2}(\rho)}{\rho}
$$

with $c(\rho)$ being the sound speed, and $p$ is the pressure that is a function of the density $\rho$. For an ideal polytropic gas, the pressure $p$ and the sound speed $c$ are given by

$$
p(\rho)=\kappa \rho^{\gamma}, \quad c^{2}(\rho)=\kappa \gamma \rho^{\gamma-1}
$$

for constants $\gamma>1$ and $\kappa>0$. Without loss of generality, we choose $\kappa=1 / \gamma$ to have

$$
i(\rho)=\frac{\rho^{\gamma-1}-1}{\gamma-1}, \quad c^{2}(\rho)=\rho^{\gamma-1} .
$$

This can be achieved by the following scaling:

$$
(\mathbf{x}, t, B) \longrightarrow\left(\alpha \mathbf{x}, \alpha^{2} t, \alpha^{-2} B\right), \quad \alpha^{2}=\kappa \gamma .
$$


Taking the limit $\gamma \rightarrow 1+$, we can also consider the case of the isothermal flow $(\gamma=1)$. For isothermal flow, (2.3) implies

$$
i(\rho)=\ln \rho, \quad c^{2}(\rho) \equiv 1 .
$$

Our goal is to find a solution $(\rho, \Phi)$ to system (2.1) (2.2) when, at $t=0$, a uniform flow in $\mathbb{R}_{+}^{2}:=\left\{x_{1} \in \mathbb{R}, x_{2}>0\right\}$ with $\left(\rho, \nabla_{\mathbf{x}} \Phi\right)=\left(\rho_{\infty}, u_{\infty}, 0\right)$ is heading to a solid ramp:

$$
W:=\left\{\mathbf{x}=\left(x_{1}, x_{2}\right): 0<x_{2}<x_{1} \tan \theta_{w}, x_{1}>0\right\} .
$$

Problem 1 (Initial-Boundary Value Problem). Seek a solution of system (2.1) -(2.2) with $B=\frac{u_{\infty}^{2}}{2}+\frac{\rho_{\infty}^{\gamma-1}-1}{\gamma-1}$ and the initial condition at $t=0$ :

$$
\left.(\rho, \Phi)\right|_{t=0}=\left(\rho_{\infty}, u_{\infty} x_{1}\right) \quad \text { for }\left(x_{1}, x_{2}\right) \in \mathbb{R}_{+}^{2} \backslash W,
$$

and with the slip boundary condition along the wedge boundary $\partial W$ :

$$
\left.\nabla_{\mathbf{x}} \Phi \cdot \boldsymbol{\nu}\right|_{\partial W \cap\left\{x_{2}>0\right\}}=0
$$

where $\boldsymbol{\nu}$ is the exterior unit normal to $\partial W$.

Notice that the initial-boundary value problem (2.1)-(2.2) with (2.4)-(2.5) is invariant under the scaling:

$$
(\mathbf{x}, t) \rightarrow(\alpha \mathbf{x}, \alpha t), \quad(\rho, \Phi) \rightarrow\left(\rho, \frac{\Phi}{\alpha}\right) \quad \text { for } \alpha \neq 0 .
$$

Thus, we seek self-similar solutions in the form of

$$
\rho(\mathbf{x}, t)=\rho(\xi, \eta), \quad \Phi(\mathbf{x}, t)=t \phi(\xi, \eta) \quad \text { for } \quad(\xi, \eta)=\frac{\mathbf{x}}{t} .
$$

Then the pseudo-potential function $\varphi=\phi-\frac{1}{2}\left(\xi^{2}+\eta^{2}\right)$ satisfies the following Euler equations for self-similar solutions:

$$
\begin{aligned}
& \operatorname{div}(\rho D \varphi)+2 \rho=0, \\
& \frac{\rho^{\gamma-1}-1}{\gamma-1}+\left(\frac{1}{2}|D \varphi|^{2}+\varphi\right)=B,
\end{aligned}
$$

where the divergence div and gradient $D$ are with respect to $(\xi, \eta)$. From this, we obtain an equation for the pseudo-potential function $\varphi(\xi, \eta)$ as follows:

$$
\operatorname{div}\left(\rho\left(|D \varphi|^{2}, \varphi\right) D \varphi\right)+2 \rho\left(|D \varphi|^{2}, \varphi\right)=0
$$

for

$$
\rho\left(|D \varphi|^{2}, \varphi\right)=\left(B_{0}-(\gamma-1)\left(\frac{1}{2}|D \varphi|^{2}+\varphi\right)\right)^{\frac{1}{\gamma-1}},
$$

where we set $B_{0}:=(\gamma-1) B+1$. Then we have

$$
c^{2}\left(|D \varphi|^{2}, \varphi\right)=B_{0}-(\gamma-1)\left(\frac{1}{2}|D \varphi|^{2}+\varphi\right) .
$$

Equation (2.8) is an equation of mixed elliptic-hyperbolic type. It is elliptic if and only if

$$
|D \varphi|<c\left(|D \varphi|^{2}, \varphi\right) .
$$


If $\rho$ is a constant, then, by (2.8) and (2.9), the corresponding pseudo-potential $\varphi$ is in the form of

$$
\varphi(\xi, \eta)=-\frac{1}{2}\left(\xi^{2}+\eta^{2}\right)+u \xi+v \eta+k
$$

for constants $u, v$, and $k$. As the incoming flow has the constant velocity $\left(u_{\infty}, 0\right)$, the corresponding pseudo-potential $\varphi_{\infty}$ has the expression of

$$
\varphi_{\infty}=-\frac{1}{2}\left(\xi^{2}+\eta^{2}\right)+u_{\infty} \xi+k_{\infty}
$$

for a constant $k_{\infty}$.

Without loss of generality by scaling, we fix $\rho_{\infty}=1$ and $M_{\infty}=\frac{u_{\infty}}{\rho_{\infty}^{(\gamma-1) / 2}}=u_{\infty}$ (Mach number of the state at infinity), and then (2.7) becomes

$$
\frac{\rho^{\gamma-1}-1}{\gamma-1}+\frac{1}{2}\left(|D \varphi|^{2}+\varphi\right)=\frac{M_{\infty}^{2}}{2}
$$

Then Problem 1 can be reformulated as the following boundary value problem in the self-similar coordinates $(\xi, \eta)$. The domain in the self-similar coordinates $(\xi, \eta)$ corresponding to $\left\{(\mathbf{x}, t): \mathbf{x} \in \mathbb{R}_{+}^{2} \backslash W, t>0\right\}$ is

$$
\Lambda:=\mathbb{R}_{+}^{2} \backslash\left\{(\xi, \eta): \eta \leq \xi \tan \theta_{w}, \xi \geq 0\right\} .
$$

Problem 2 (Boundary Value Problem). Seek a solution $\varphi$ of equation (2.8) in the self-similar domain $\Lambda$ with the slip boundary condition:

$$
D \varphi \cdot \boldsymbol{\nu}_{w}=0 \quad \text { on } \Gamma_{\text {wedge }}=\left\{(\xi, \eta): \eta=\xi \tan \theta_{w}, \xi>0\right\},
$$

where $\boldsymbol{\nu}_{w}$ is the exterior unit normal to the boundary of the wedge $\Gamma_{w e d g e}$.

In particular, we seek a weak solution of Problem 2 such that it contains a straight weak oblique shock attached to the tip of the wedge, and the oblique shock is connected to a normal shock through a curved shock as shown in Figure 1 .

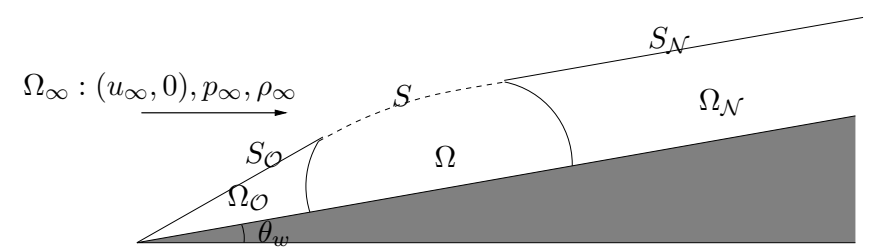

FIGURE 1. Weak shock solutions in the self-similar coordinates

A shock is a curve across which $D \varphi$ is discontinuous. If $\Omega^{+}$and $\Omega^{-}\left(:=\Omega \backslash \overline{\Omega^{+}}\right)$ are two nonempty open subsets of $\Omega \subset \mathbb{R}^{2}$, and $S:=\partial \Omega^{+} \cap \Omega$ is a $C^{1}$-curve where $D \varphi$ has a jump, then $\varphi \in W_{l o c}^{1,1} \cap C^{1}\left(\Omega^{ \pm} \cup S\right) \cap C^{2}\left(\Omega^{ \pm}\right)$is a global weak solution of (2.8) in $\Omega$ if and only if $\varphi$ is in $W_{l o c}^{1, \infty}(\Omega)$ and satisfies equation (2.8) and the Rankine-Hugoniot condition on $S$ :

$$
\left[\rho\left(|D \varphi|^{2}, \varphi\right) D \varphi \cdot \boldsymbol{\nu}\right]_{S}=0
$$

where $[F]_{S}$ is defined by

$$
[F(\xi, \eta)]_{S}:=\left.F(\xi, \eta)\right|_{\overline{\Omega^{-}}}-\left.F(\xi, \eta)\right|_{\overline{\Omega^{+}}} \quad \text { for } \quad(\xi, \eta) \in S .
$$


Note that the condition $\varphi \in W_{l o c}^{1, \infty}(\Omega)$ requires

$$
[\varphi]_{S}=0 .
$$

In Figure 1, by (2.12) and (2.16), the pseudo-potentials $\varphi_{\mathcal{O}}$ and $\varphi_{\mathcal{N}}$ below $S_{\mathcal{O}}$ and $S_{\mathcal{N}}$ are respectively in the form of

$$
\begin{aligned}
& \varphi_{\mathcal{O}}=-\frac{1}{2}\left(\xi^{2}+\eta^{2}\right)+u_{\mathcal{O}} \xi+v_{\mathcal{O}} \eta+k_{\mathcal{O}}, \\
& \varphi_{\mathcal{N}}=-\frac{1}{2}\left(\xi^{2}+\eta^{2}\right)+u_{\mathcal{N}} \xi+v_{\mathcal{N}} \eta+k_{\mathcal{N}}
\end{aligned}
$$

for constants $u_{\mathcal{O}}, v_{\mathcal{O}}, u_{\mathcal{N}}, v_{\mathcal{N}}, k_{\mathcal{O}}$, and $k_{\mathcal{N}}$. Then it follows from (2.9) and (2.17) that the corresponding densities $\rho_{\mathcal{O}}$ and $\rho_{\mathcal{N}}$ below $S_{\mathcal{O}}$ and $S_{\mathcal{N}}$ are constants, respectively.

Given $M_{\infty}>1$, we obtain $\left(u_{\mathcal{O}}, v_{\mathcal{O}}\right)$ and $\rho_{\mathcal{O}}$ by using the shock polar curve for steady potential flow in Figure 2, In Figure 2, let $\theta_{\text {sonic }}$ be the wedge angle such that the line $\frac{v}{u}=\tan \theta_{\text {sonic }}$ intersects with the shock polar curve at a point on the circle of radius 1 . For a wedge angle $\theta_{w} \in\left(0, \theta_{\text {sonic }}\right)$, the line $v=u \tan \theta_{w}$ and the shock polar curve intersect at a point $\left(u_{\mathcal{O}}, v_{\mathcal{O}}\right)$ with $\sqrt{u_{\mathcal{O}}^{2}+v_{\mathcal{O}}^{2}}>1$ and $u_{\mathcal{O}}<u_{\infty}$. The intersection $\left(u_{\mathcal{O}}, v_{\mathcal{O}}\right)$ indicates the velocity for steady potential flow behind an oblique shock $S_{\mathcal{O}}$ attached to the tip of the wedge angle $\theta_{w}$. Since the strength of the shock $S_{\mathcal{O}}$ is relatively weak compared to the other shock given by the other intersection point on the shock polar curve, $S_{\mathcal{O}}$ is called a weak shock. We also note that the states on both sides of $S_{\mathcal{O}}$ are supersonic, and such states $\left(u_{\mathcal{O}}, v_{\mathcal{O}}\right)$ smoothly depend on $u_{\infty}$ and $\theta_{w}$. Once $\left(u_{\mathcal{O}}, v_{\mathcal{O}}\right)$ is determined, then, by (2.13), the

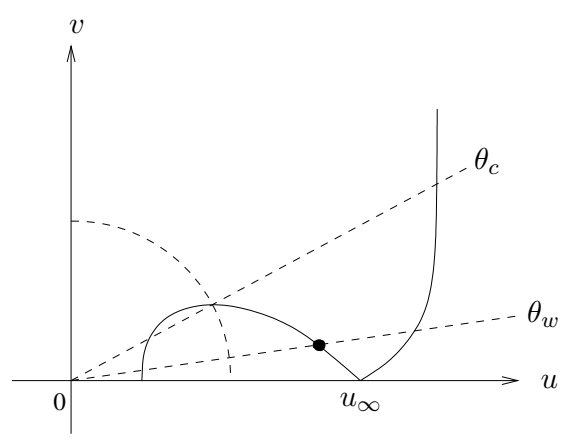

FiguRE 2. Shock polar

corresponding density $\rho_{\mathcal{O}}$ is given by

$$
\rho_{\mathcal{O}}^{\gamma-1}=1+\frac{\gamma-1}{2}\left(u_{\infty}^{2}-u_{\mathcal{O}}^{2}-v_{\mathcal{O}}^{2}\right) .
$$

To find a curved shock $S$ connecting the oblique shock $S_{\mathcal{O}}$ with the normal shock $S_{\mathcal{N}}$ in Figure 1, it is more convenient to use a coordinate system in which the boundary of wedge $\Gamma_{\text {wedge }}$ is the same for all $\theta_{w} \in\left(0, \theta_{\text {sonic }}\right)$. For that reason, we introduce a new coordinate system $\left(\xi^{\prime}, \eta^{\prime}\right)$ defined by

$$
\left(\begin{array}{c}
\xi^{\prime} \\
\eta^{\prime}
\end{array}\right)=\left(\begin{array}{cc}
\cos \theta_{w} & \sin \theta_{w} \\
-\sin \theta_{w} & \cos \theta_{w}
\end{array}\right)\left(\begin{array}{l}
\xi \\
\eta
\end{array}\right)-\left(\begin{array}{c}
u_{\mathcal{O}} \cos \theta_{w} \\
0
\end{array}\right) .
$$


In other words, $\left(\xi^{\prime}, \eta^{\prime}\right)$ is obtained by rotating $(\xi, \eta)$ and translating the self-similar plane. Denoting as $(\xi, \eta)$ for $\left(\xi^{\prime}, \eta^{\prime}\right), \varphi_{\infty}$ and $\varphi_{\mathcal{O}}$ defined by (2.12) and (2.17) are expressed as

$$
\begin{aligned}
& \varphi_{\infty}=-\frac{1}{2}\left(\xi^{2}+\eta^{2}\right)-\eta u_{\infty} \sin \theta_{w}+k_{\infty} \\
& \varphi_{\mathcal{O}}=-\frac{1}{2}\left(\xi^{2}+\eta^{2}\right)+\widetilde{u_{\mathcal{O}}} \xi+k_{\mathcal{O}}
\end{aligned}
$$

with

$$
\widetilde{u_{\mathcal{O}}}=\sqrt{u_{\mathcal{O}}^{2}+v_{\mathcal{O}}^{2}}-u_{\infty} \cos \theta_{w}
$$

for constants $k_{\infty}$ and $k_{\mathcal{O}}$ different from (2.12) and (2.17). For simplicity, we will write $\widetilde{u_{\mathcal{O}}}$ as $u_{\mathcal{O}}$ hereafter. Without loss of generality, we choose $k_{\infty}=0$. Set

$$
v_{\infty}:=u_{\infty} \sin \theta_{w},
$$

and let $\beta$ be the angle between the oblique shock $S_{\mathcal{O}}$ and wedge $\Gamma_{\text {wedge }}$. We use the parameters $\left(v_{\infty}, \beta\right)$ instead of $\left(u_{\infty}, \theta_{w}\right)$ to compute the normal shock $S_{\mathcal{N}}$ and the state behind it.

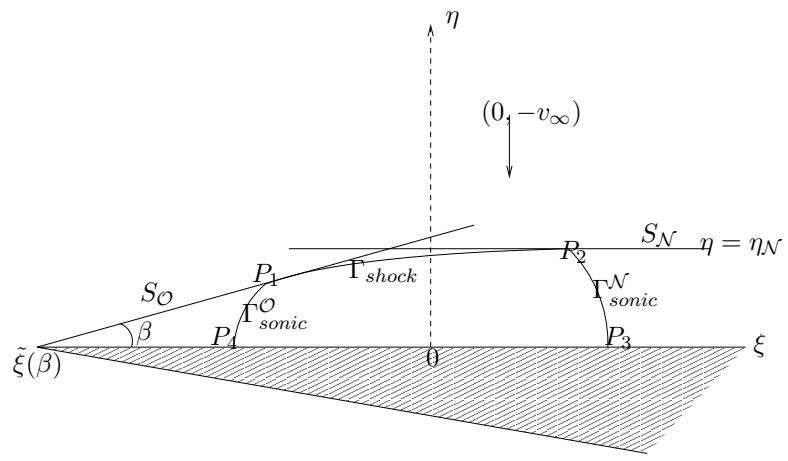

FIGURE 3. Weak shock solutions on the self-similar plane

By (2.16), the definition of $\varphi_{\infty}$ given by (2.20) with $k_{\infty}=0$, and by the slip boundary condition (2.14), if we let $S_{\mathcal{N}}=\left\{\eta=\eta_{\mathcal{N}}\right\}$ for a constant $\eta_{\mathcal{N}}$, then we have

$$
\varphi_{\mathcal{N}}=-\frac{1}{2}\left(\xi^{2}+\eta^{2}\right)-v_{\infty} \eta_{\mathcal{N}}
$$

By (2.15) and (2.13), the density $\rho_{\mathcal{N}}$ and constant $\eta_{\mathcal{N}}$ satisfy

$$
\begin{aligned}
& \eta_{\mathcal{N}}=\frac{v_{\infty}}{\rho_{\mathcal{N}}-1} \\
& \frac{\rho_{\mathcal{N}}^{\gamma-1}-1}{\gamma-1}=\frac{1}{2} v_{\infty}^{2}+\eta_{\mathcal{N}} v_{\infty} .
\end{aligned}
$$

We note that $0<\eta_{\mathcal{N}}<c_{\mathcal{N}}$ for $c_{\mathcal{N}}=\rho_{\mathcal{N}}^{(\gamma-1) / 2}$.

Fix $\beta \in\left(0, \frac{\pi}{2}\right)$, consider an oblique shock $S_{\mathcal{O}}$ of the angle $\beta$ from the $\xi$-axis, and let $\varphi_{\mathcal{O}}$ be the corresponding pseudo-potential below $S_{\mathcal{O}}$. Let $\left(\xi_{\mathcal{O}}, \eta_{\mathcal{O}}\right)$ be the intersection $P_{1}$ of $S_{\mathcal{O}}$ and the sonic circle $B_{c_{\mathcal{O}}}\left(u_{\mathcal{O}}, 0\right)$ for the state in $\Omega^{\mathcal{O}}$ (see Figure 
1). There are two intersection points, and we denote $P_{1}$ the point with the smaller value of the $\xi$-coordinate (see Figure 3 ). Given $v_{\infty}>0$ and $\gamma \geq 1, \eta_{\mathcal{O}}$ satisfies

$$
\frac{\partial \eta_{\mathcal{O}}}{\partial \beta}<0 \quad \text { for all } \beta \in\left(0, \frac{\pi}{2}\right) \text {. }
$$

Therefore, the set $I_{v_{\infty}}:=\left\{\beta \in\left(0, \frac{\pi}{2}\right): 0<\eta_{\mathcal{O}}(\beta)<\infty\right\}$ is connected and, furthermore, there exists $\beta_{\text {sonic }} \in\left(0, \frac{\pi}{2}\right]$ satisfying

$$
I_{v_{\infty}}=\left(0, \beta_{\text {sonic }}\right)
$$

and such $\beta_{\text {sonic }}$ depends smoothly on $v_{\infty}$ and $\gamma$.

Given $\gamma \geq 1$, we note that $\theta_{\text {sonic }}$ on the shock polar curve in Figure 2 depends on $u_{\infty}$, while $\beta_{\text {sonic }}$ depends on $v_{\infty}$, so that we write as $\theta_{\text {sonic }}\left(u_{\infty}\right)$ and $\beta_{\text {sonic }}\left(v_{\infty}\right)$, respectively. Define two parameter sets $\mathfrak{P}_{1}$ and $\mathfrak{P}_{2}$ by

$$
\begin{aligned}
& \mathfrak{P}_{1}:=\bigcup_{u_{\infty}>1}\left\{u_{\infty}\right\} \times\left(0, \theta_{\text {sonic }}\left(u_{\infty}\right)\right), \\
& \mathfrak{P}_{2}:=\bigcup_{v_{\infty}>0}\left\{v_{\infty}\right\} \times\left(0, \beta_{\text {sonic }}\left(v_{\infty}\right)\right) .
\end{aligned}
$$

Then we have the following lemma.

Lemma 2.1. For any $\gamma \geq 1$, there exists a one-to-one and onto correspondence between $\mathfrak{P}_{1}$ and $\mathfrak{P}_{2}$.

Remark 2.2 (Normal shock: $\beta=0$ ). For fixed $\gamma \geq 1$ and $v_{\infty}>0$, the state of $\beta=0$ is a normal shock, and this corresponds to the state of $\left(u_{\infty}, \theta_{w}\right)=(\infty, 0)$. Even though $\beta=0$ is nonphysical, we will put the case of $\beta=0$ in our consideration as it is useful in applying the Leray-Schauder degree theorem to establish Theorem 1 stated in Section 3.

For a fixed $\left(v_{\infty}, \beta\right) \in \mathfrak{P}_{2}$, if we can prove the existence of a curved shock $\Gamma_{\text {shock }}$ to connect $S_{\mathcal{O}}$ with $S_{\mathcal{N}}$ and the existence of corresponding pseudo-potential below $\Gamma_{\text {shock}}$, then, by Lemma2.1, the existence of a curved shock and the pseudo-potential below the shock for $\left(u_{\infty}, \theta_{w}\right) \in \mathfrak{P}_{1}$ corresponding to the fixed $\left(v_{\infty}, \beta\right)$ automatically follows. By taking $\left(v_{\infty}, \beta\right)$ as a parameter instead of $\left(u_{\infty}, \theta_{w}\right)$, it is advantageous that the boundary of a wedge is always on the $\xi$-axis. As $\left(v_{\infty}, \beta\right)$ is the parameter of our problem, we write $\varphi_{\infty}$ in (2.20) as

$$
\varphi_{\infty}=-\frac{1}{2}\left(\xi^{2}+\eta^{2}\right)-v_{\infty} \eta
$$

Since the states below $S_{\mathcal{O}}$ and $S_{\mathcal{N}}$ are given by $\varphi_{\mathcal{O}}$ and $\varphi_{\mathcal{N}}$, in order to solve Problem 2, it suffices to solve the following problem.

Problem 3 (Free Boundary Problem). Find a curved shock $\Gamma_{\text {shock }}$ and a function $\varphi$ defined in the region $\Omega$, enclosed by $\Gamma_{\text {shock }}, \Gamma_{\text {sonic }}^{\mathcal{O}}, \Gamma_{\text {sonic }}^{\mathcal{N}}$, and $\{\eta=0\}$, such that $\varphi$ satisfies

(i) Equation (2.8) in $\Omega$;

(ii) $\varphi=\varphi_{\infty}, \rho D \varphi \cdot \boldsymbol{\nu}_{s}=D \varphi_{\infty} \cdot \boldsymbol{\nu}_{s}$ on $\Gamma_{\text {shock }}$;

(iii) $\varphi=\varphi_{\beta}, D \varphi=D \varphi_{\beta}$ on $\Gamma_{\text {sonic }}^{\mathcal{O}} \cup \Gamma_{\text {sonic }}^{\mathcal{N}}$ for $\varphi_{\beta}:=\max \left(\varphi_{\mathcal{O}}, \varphi_{\mathcal{N}}\right)$;

(iv) $\partial_{\eta} \varphi=0$ on $\Gamma_{\text {wedge }}$, 
where $\boldsymbol{\nu}_{s}$ is the interior unit normal on $\Gamma_{\text {shock. }}$.

Let $\varphi$ be a solution of Problem 3 with a shock $\Gamma_{\text {shock}}$. Moreover, assume that $\varphi \in C^{1}(\bar{\Omega})$, and $\Gamma_{\text {shock }}$ is a $C^{1}$-curve up to its endpoints. To obtain a solution of Problem 2 from $\varphi$, we divide the half-plane $\{\eta \geq 0\}$ into four separate regions. Let $\Omega_{E}$ be the unbounded domain below the curve $\overline{S_{\mathcal{O}} \cup \Gamma_{\text {shock }} \cup S_{\mathcal{N}}}$ in $\{\eta \geq 0\}$ (see Figure 3). In $\Omega_{E}$, let $\Omega_{\mathcal{O}}$ be the bounded open domain enclosed by $S_{\mathcal{O}}, \Gamma_{\text {sonic }}^{\mathcal{O}}$, and $\{\eta=0\}$. We set $\Omega_{\mathcal{N}}:=\Omega_{E} \backslash \overline{\left(\Omega_{\mathcal{O}} \cup \Omega\right)}$. Define a function $\varphi_{*}$ in $\{\eta \geq 0\}$ by

$$
\varphi_{*}= \begin{cases}\varphi_{\infty} & \text { in }\{\eta \geq 0\} \backslash \Omega_{E}, \\ \varphi_{\mathcal{O}} & \text { in } \Omega_{\mathcal{O}}, \\ \varphi & \text { in } \Gamma_{\text {sonic }}^{\mathcal{O}} \cup \Omega \cup \Gamma_{\text {sonic }}^{\mathcal{N}} \\ \varphi_{\mathcal{N}} & \text { in } \Omega_{\mathcal{N}} .\end{cases}
$$

By (2.16) and (iii) of Problem 3, $\varphi_{*}$ is continuous in $\{\eta \geq 0\}$ and is $C^{1}$ in $\overline{\Omega_{E}}$. In particular, $\varphi_{*}$ is $C^{1}$ across $\Gamma_{\text {sonic }}^{\mathcal{O}} \cup \Gamma_{\text {sonic }}^{\mathcal{N}}$. Moreover, using (i)-(iii) of Problem 3, we obtain that $\varphi_{*}$ is a weak solution of equation (2.8) in $\{\eta>0\}$. Applying the inverse coordinate transformation of (2.19) to $\varphi_{*}$, we obtain a solution of Problem 2 ,

\section{Results}

In this section we first present the known result, Elling-Liu's theorem, in [13, and then we describe our new results for Problem 3, the Prandtl-Meyer problem.

3.1. Known Result. Elling and Liu in [13] have established the following theorem.

Elling-Liu's Theorem (Theorem 1 in [13]). Given $\left(v_{\infty}, \beta\right) \in \mathfrak{P}_{2}$, let $L$ be the line segment connecting $P_{1}$ with $P_{2}$ in Figure 3. If $L$ does not intersect with $B_{1}\left(0,-v_{\infty}\right)$, then there exists a weak solution to Problem 2 with structure (2.29).

If $v_{\infty}>1$, since $B_{1}\left(0,-v_{\infty}\right) \subset\{\eta<0\}$, the assumption in the theorem above holds true. On the other hand, for $v_{\infty}<1$, there exists a set of parameters $\left(v_{\infty}, \beta\right) \in$ $\mathfrak{P}_{2}$ for which the assumption no long holds. This can be shown as follows:

Fix $\gamma \geq 1$ and $v_{\infty}>0$. Let $L_{\infty}$ be the line with positive slope such that $L_{\infty}$ is tangent to the sonic circle $B_{1}\left(0,-v_{\infty}\right)$ of the incoming state and that it passes through the point $P_{2}=\left(\xi_{\mathcal{N}}, \eta_{\mathcal{N}}\right)=\overline{\Gamma_{\text {sonic }}^{\mathcal{N}}} \cap S_{\mathcal{N}}$. For some $v_{\infty}>0$, there may be two such tangent lines. In that case, we choose the one with the smaller slope. We also fix $\beta \in\left(0, \beta_{\text {sonic }}\left(v_{\infty}\right)\right)$. Let $L_{\mathcal{O}}$ be the line segment connecting $P_{1}:=\left(\xi_{\mathcal{O}}, \eta_{\mathcal{O}}\right)=$ $\overline{\Gamma_{\text {sonic }}^{\mathcal{O}}} \cap S_{\mathcal{O}}$ with $P_{2}$ (see Figure 4).

Let $\tan \theta_{\mathcal{O}}$ and $\tan \theta_{\infty}$ be the slopes of the lines $L_{\mathcal{O}}$ and $L_{\infty}$, respectively. If $\theta_{\mathcal{O}}>\theta_{\infty}$, then the line $L_{\mathcal{O}}$ intersects with the sonic circle $B_{1}\left(0,-v_{\infty}\right)$. On the other hand, if $\theta_{\mathcal{O}}<\theta_{\infty}$, then $L_{\mathcal{O}}$ has no intersection with $B_{1}\left(0,-v_{\infty}\right)$. Define

$$
F(\beta):=\tan \theta_{\mathcal{O}}-\tan \theta_{\infty}
$$

Then, we have the following proposition. 


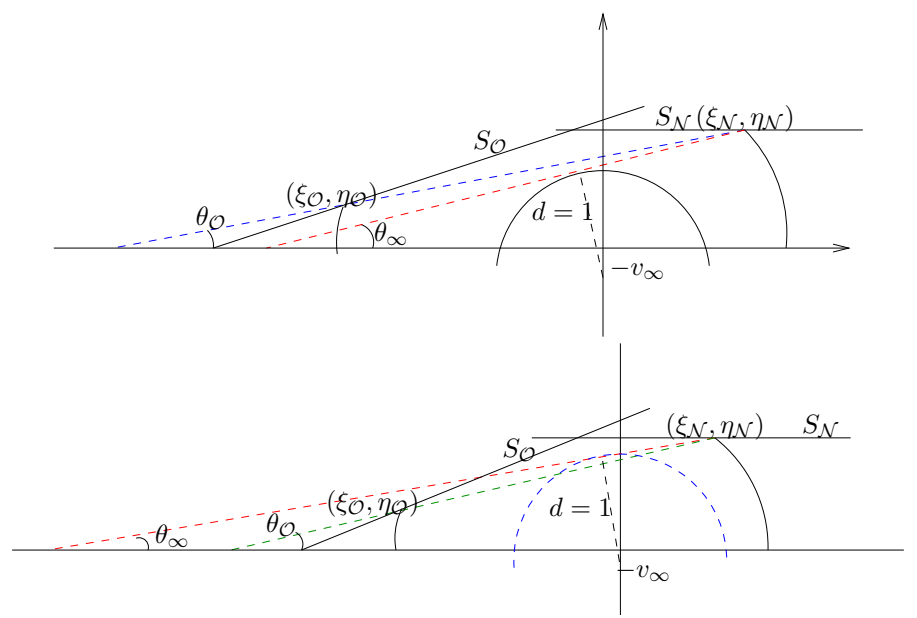

Figure 4. Top: $\theta_{\mathcal{O}}<\theta_{\infty} ; \quad$ Bottom: $\theta_{\mathcal{O}}>\theta_{\infty}$

Proposition 3.1. For any given $\gamma \geq 1$, there exists $v_{*}>0$ depending on $\gamma$ so that, wherever $v_{\infty} \in\left(0, v_{*}\right)$, there exists $\hat{\beta}=\hat{\beta}\left(v_{\infty}\right) \in\left(0, \beta_{\text {sonic }}\left(v_{\infty}\right)\right)$ satisfying

$$
\begin{array}{ll}
F(\beta) \leq 0 & \text { for } \beta \in[0, \hat{\beta}], \\
F(\beta)>0 & \text { for } \beta \in\left(\hat{\beta}, \beta_{\text {sonic }}\right) .
\end{array}
$$

According to Proposition 3.1 , there exists a subset of $\mathfrak{P}_{2}$ for which [13, Theorem 1] does not apply.

3.2. New Results. Our new results provide not only the existence of a global solution to Problem 3 for all $\left(v_{\infty}, \beta\right)$ in $\mathfrak{P}_{2}$, but also the higher regularity of the solution in pseudo-subsonic regions. Therefore, we achieve the existence of a selfsimilar weak solution with higher regularity to Problem 1 for all $\left(u_{\infty}, \theta_{w}\right)$ in $\mathfrak{P}_{1}$.

For any fixed $\left(v_{\infty}, \beta\right) \in \mathfrak{P}_{2}$, the oblique shock $S_{\mathcal{O}}$ of the slope $\tan \beta$, the normal shock $S_{\mathcal{N}}$, and the corresponding pseudo-potentials $\varphi_{\mathcal{O}}$ and $\varphi_{\mathcal{N}}$ below $S_{\mathcal{O}}$ and $S_{\mathcal{N}}$ are uniquely computed. Let $(\tilde{\xi}(\beta), 0)$ be the $\xi$-intercept of $S_{\mathcal{O}}$. Then we have

$$
S_{\mathcal{O}} \subset\left\{\left(\xi, f_{\mathcal{O}}(\xi)\right): f_{\mathcal{O}}(\xi)=\tan \beta(\xi-\tilde{\xi}(\beta)), \xi \in \mathbb{R}\right\} .
$$

Denoting $P_{1}=\left(\xi_{\mathcal{O}}, \eta_{\mathcal{O}}\right)$ and $P_{2}=\left(\xi_{\mathcal{N}}, \eta_{\mathcal{N}}\right)$ in Figure 3, we have

$$
S_{\mathcal{O}}=\left\{(\xi, \eta): \tilde{\xi}(\beta) \leq \xi \leq \xi_{\mathcal{O}}, \eta=f_{\mathcal{O}}(\xi)\right\}, \quad S_{\mathcal{N}}=\left\{\left(\xi, \eta_{\mathcal{N}}\right): \xi \geq \xi_{\mathcal{N}}\right\} .
$$

Theorem 1. For any given $\gamma \geq 1$ and $\left(v_{\infty}, \beta\right) \in \mathfrak{P}_{2}$, there exists a global weak solution $\varphi$ of Problem 3 satisfying the following properties:

(i) There exists a shock curve $\Gamma_{\text {shock }}$ with endpoints $P_{1}=\left(\xi_{\mathcal{O}}, \eta_{\mathcal{O}}\right)$ and $P_{2}=$ $\left(\xi_{\mathcal{N}}, \eta_{\mathcal{N}}\right)$ such that

$-\Gamma_{\text {shock }}$ satisfies $\Gamma_{\text {shock }} \subset\left(\mathbb{R}_{+}^{2} \backslash \overline{B_{1}\left(0,-v_{\infty}\right)}\right) \cap\left\{\xi_{\mathcal{O}} \leq \xi \leq \xi_{\mathcal{N}}\right\}$;

- $\Gamma_{\text {shock }}$ is $C^{3}$ in its relative interior: For any $P \in \Gamma_{\text {shock }} \backslash\left\{P_{1}, P_{2}\right\}$, there exist $r>0$ and $f \in C^{3}(\mathbb{R})$ such that

$$
\Gamma_{\text {shock }} \cap B_{r}(P)=\{(\xi, \eta): \eta=f(\xi)\} \cap B_{r}(P) ;
$$


- The curve $\overline{S_{\mathcal{O}} \cup \Gamma_{\text {shock }} \cup S_{\mathcal{N}}}$ is $C^{1}$, including at the points $P_{1}$ and $P_{2}$;

$-\Gamma_{\text {shock }}, \Gamma_{\text {sonic }}^{\mathcal{N}}, \Gamma_{\text {sonic }}^{\mathcal{O}}$, and $\Gamma_{\text {wedge }}:=\left\{(\xi, 0): u_{\mathcal{O}}-c_{\mathcal{O}} \leq \xi \leq c_{\mathcal{N}}\right\}$ do not have common points except at their end points. Thus, $\Gamma_{\text {shock }} \cup \Gamma_{\text {sonic }}^{\mathcal{N}} \cup$ $\Gamma_{\text {sonic }}^{\mathcal{O}} \cup \Gamma_{\text {wedge }}$ is a closed curve without self-intersections. Denote by $\Omega$ the open and bounded domain enclosed by this closed curve.

(ii) $\varphi$ satisfies $\varphi \in C^{3}\left(\bar{\Omega} \backslash\left(\Gamma_{\text {sonic }}^{\mathcal{O}} \cup \Gamma_{\text {sonic }}^{\mathcal{N}}\right)\right) \cap C^{1}(\bar{\Omega})$.

(iii) Equation (2.8) is strictly elliptic in $\bar{\Omega} \backslash\left(\Gamma_{\text {sonic }}^{\mathcal{N}} \cup \Gamma_{\text {sonic }}^{\mathcal{O}}\right)$.

(iv) $\max \left(\varphi_{\mathcal{O}}, \varphi_{\mathcal{N}}\right) \leq \varphi \leq \varphi_{\infty}$ in $\Omega$.

(v) $\partial_{-\hat{\xi}}\left(\varphi_{\infty}-\varphi\right) \leq 0$ and $\partial_{\hat{e}_{S_{\mathcal{O}}}}\left(\varphi_{\infty}-\varphi\right) \leq 0$ in $\Omega$ for

If $\varphi$ is a weak solution satisfying properties (i)-(v) of Theorem 1, then $\varphi$ and $\Gamma_{\text {shock }}$ satisfy additional regularity properties.

Theorem 2. Given $\gamma \geq 1$ and $\left(v_{\infty}, \beta\right) \in \mathfrak{P}_{2}$, let $\varphi$ be a weak solution of Problem 1 satisfying properties (i)-(v) of Theorem 1. Then the following properties hold:

(i) The curve $\overline{S_{\mathcal{O}} \cup \Gamma_{\text {shock }} \cup S_{\mathcal{N}}}$ is $C^{2, \alpha}$ for any $\alpha \in\left[0, \frac{1}{2}\right)$, including at the points $P_{1}$ and $P_{2}$. Moreover, $\Gamma_{\text {shock }}$ is $C^{\infty}$ in its relative interior.

(ii) $\varphi \in C^{\infty}\left(\bar{\Omega} \backslash\left(\Gamma_{\text {sonic }}^{\mathcal{O}} \cup \Gamma_{\text {sonic }}^{\mathcal{N}}\right)\right) \cap C^{1,1}(\bar{\Omega})$.

(iii) For a constant $\sigma>0$ and a set $\mathcal{D}$ given by

$$
\mathcal{D}=\left\{(\xi, \eta): \max \left(\varphi_{\mathcal{O}}(\xi, \eta), \varphi_{\mathcal{N}}(\xi, \eta)\right)<\varphi_{\infty}(\xi, \eta), \eta>0\right\},
$$

define

$$
\begin{aligned}
& \mathcal{D}_{\sigma}^{\mathcal{N}}=\mathcal{D} \cap\left\{(\xi, \eta): \operatorname{dist}\left((\xi, \eta), \Gamma_{\text {sonic }}^{\mathcal{N}}\right)<\sigma\right\} \cap B_{c_{\mathcal{N}}}(0,0), \\
& \mathcal{D}_{\sigma}^{\mathcal{O}}=\mathcal{D} \cap\left\{(\xi, \eta): \operatorname{dist}\left((\xi, \eta), \Gamma_{\text {sonic }}^{\mathcal{O}}\right)<\sigma\right\} \cap B_{c_{\mathcal{O}}}\left(u_{\mathcal{O}}, 0\right)
\end{aligned}
$$

for $c_{\mathcal{N}}=\rho_{\mathcal{N}}^{(\gamma-1) / 2}$ and $c_{\mathcal{O}}=\rho_{\mathcal{O}}^{(\gamma-1) / 2}$. Then, for any $\alpha \in(0,1)$ and any given $\left.\left(\xi_{0}, \eta_{0}\right) \in \overline{\left(\overline{\Gamma_{\text {sonic }}^{\mathcal{O}}}\right.} \cup \overline{\Gamma_{\text {sonic }}^{\mathcal{N}}}\right) \backslash\left\{P_{1}, P_{2}\right\}$, there exists $K<\infty$ depending only on $\gamma, v_{\infty}, \varepsilon_{0}, \alpha,\|\varphi\|_{C^{1,1}\left(\Omega \cap\left(\mathcal{D}_{\varepsilon_{0}}^{\mathcal{O}} \cup \mathcal{D}_{\varepsilon_{0}}^{\mathcal{N}}\right)\right)}$, and $d=\operatorname{dist}\left(\left(\xi_{0}, \eta_{0}\right), \Gamma_{\text {shock }}\right)$ so that there holds

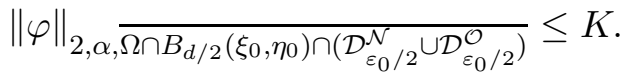

(iv) For any $\left(\xi_{0}, \eta_{0}\right) \in\left(\Gamma_{\text {sonic }}^{\mathcal{O}} \cup \Gamma_{\text {sonic }}^{\mathcal{N}}\right) \backslash\left\{P_{1}, P_{2}\right\}$,

$$
\lim _{\substack{(\xi, \eta) \rightarrow\left(\xi_{0}, \eta_{0}\right) \\(\xi, \eta) \in \Omega}}\left(D_{r r} \varphi-D_{r r} \max \left(\varphi_{\mathcal{N}}, \varphi_{\mathcal{O}}\right)\right)=\frac{1}{\gamma+1}
$$

where $r=\sqrt{\xi^{2}+\eta^{2}}$ near $\Gamma_{\text {sonic }}^{\mathcal{N}}$, and $r=\sqrt{\left(\xi-u_{\mathcal{O}}\right)^{2}+\eta^{2}}$ near $\Gamma_{\text {sonic }}^{\mathcal{O}}$.

(v) The limits $\lim _{\substack{(,, \eta) \rightarrow P_{1} \\(\xi, \eta) \in \Omega}} D^{2} \varphi$ and $\lim _{\substack{(\xi, \eta) \rightarrow P_{2} \\(\xi, \eta) \in \Omega}} D^{2} \varphi$ do not exist.

Remark 3.2. Assertion (iii) of Theorem 2 says that $\varphi$ in $\Omega$ is $C^{2, \alpha}$ up to the sonic arcs $\Gamma_{\text {sonic }}^{\mathcal{O}}$ and $\Gamma_{\text {sonic }}^{\mathcal{N}}$ away from the points $P_{1}$ and $P_{2}$. Assertion (ii) of Theorem 2 combined with (iii) of Problem 3 imply that the function $\varphi_{*}$ in (2.29) is $C^{1,1}$ across the sonic arcs $\Gamma_{\text {sonic }}^{\mathcal{N}}$ and $\Gamma_{\text {sonic }}^{\mathcal{O}}$. Assertion (iv) of Theorem 2 implies that the function $\varphi_{*}$ is not $C^{2}$ across the sonic arcs $\Gamma_{\text {sonic }}^{\mathcal{N}}$ and $\Gamma_{\text {sonic }}^{\mathcal{O}}$ since there is a jump of second derivative of $\varphi$, which implies that the $C^{1,1}$-regularity is optimal. 
Remark 3.3 (Weak solutions of Problem 3 for $\beta=0$ ). For $\beta=0, \varphi=\varphi_{\mathcal{N}}$ is the unique weak solution of Problem 3 satisfying properties (i)-(v) of Theorem 1, where $\varphi_{\mathcal{N}}$ is defined by (2.23).

The following theorem easily follows from Theorem 1, Lemma 2.1, and the argument after (2.29).

Theorem 3. For any given $\gamma \geq 1$ and any $\left(u_{\infty}, \theta\right) \in \mathfrak{P}_{1}$, Problem 2 has a global weak solution $\varphi_{*}$ of the structure as in Figure 1 with $\varphi_{*}$ being continuous in $\Lambda$ and $C^{1}$ across $\Gamma_{\text {sonic }}^{\mathcal{O}} \cup \Gamma_{\text {sonic }}^{\mathcal{N}}$.

\section{Overview of the proof of Theorem 1}

The key to establish Theorem 1 is in the monotonicity properties of $\varphi_{\infty}-\varphi$ for a weak solution $\varphi$ of Problem 3. It implies that the shock is Lipschitz graph in a cone of directions and thus fixes geometry of the problem, among other consequences. Another key property is following. Fix $\gamma \geq 1, v_{\infty}>0$, and $\beta_{*} \in\left(0, \beta_{\text {sonic }}\left(v_{\infty}\right)\right)$. Then there exists a constant $C>0$ depending only on $\gamma, v_{\infty}$, and $\beta_{*}$ such that, for any $\beta \in\left(0, \beta_{*}\right]$, a corresponding weak solution $\varphi$ with properties (i)-(v) in Theorem 1 satisfies

$$
\operatorname{dist}\left(\Gamma_{\text {shock }}, B_{1}\left(0,-v_{\infty}\right)\right) \geq \frac{1}{C}>0 .
$$

This inequality plays an essential role to achieve the ellipticity of equation (2.8) in $\Omega$. Once the ellipticity is achieved, then we obtain various apriori estimates of $\varphi$ so that we can employ a degree theory to establish the existence of a weak solution for all $\beta \in\left(0, \beta_{*}\right]$. Since the choice of $\beta_{*}$ is arbitrary in $\left(0, \beta_{\text {sonic }}\left(v_{\infty}\right)\right)$, the existence of a weak solution for any $\beta \in\left(0, \beta_{\text {sonic }}\left(v_{\infty}\right)\right)$ can be established.

For the rest of this paper, we outline the proof of Theorem 1 .

4.1. Monotonicity Property and Its Consequences. Given $v_{\infty}>0$ and $\beta \in$ $\left(0, \beta_{*}\right]$, set $\hat{e}_{S_{\mathcal{O}}}:=(\cos \beta, \sin \beta)$. Then $\hat{e}_{S_{\mathcal{O}}}$ is a unit tangent to $S_{\mathcal{O}}$ and satisfies $\hat{e}_{S_{\mathcal{O}}} \cdot \hat{\xi}>0$.

Lemma 4.1. For a fixed $\left(v_{\infty}, \beta\right) \in \mathfrak{P}_{2}$, if $\varphi$ is a weak solution with properties (i)-(v) in Theorem 1, then it satisfies

$$
\begin{aligned}
& \partial_{\hat{e}_{S_{\mathcal{O}}}}\left(\varphi_{\infty}-\varphi\right)<0 \quad \text { in } \bar{\Omega} \backslash \overline{\Gamma_{\text {sonic }}^{\mathcal{O}}}, \quad \partial_{\xi}\left(\varphi_{\infty}-\varphi\right)>0 \quad \text { in } \bar{\Omega} \backslash \overline{\Gamma_{\text {sonic }}^{\mathcal{N}}}, \\
& \partial_{\xi}\left(\varphi-\varphi_{\mathcal{N}}\right) \leq 0, \quad \partial_{\eta}\left(\varphi-\varphi_{\mathcal{N}}\right) \leq 0 \quad \text { in } \bar{\Omega}, \\
& \partial_{\hat{e}_{S_{\mathcal{O}}}}\left(\varphi-\varphi_{\mathcal{O}}\right) \geq 0, \quad \partial_{\eta}\left(\varphi-\varphi_{\mathcal{O}}\right) \leq 0 \quad \text { in } \bar{\Omega} .
\end{aligned}
$$

For $\beta \in\left(0, \beta_{*}\right]$, and two unit vectors $\hat{e}_{S_{\mathcal{O}}}$ and $-\hat{\xi}$ in $\mathbb{R}^{2}$, define

$$
\text { Cone }\left(\hat{e}_{S_{\mathcal{O}}},-\hat{\xi}\right):=\left\{a_{1} \hat{e}_{S_{\mathcal{O}}}+a_{2}(-\hat{\xi}): a_{1}, a_{2} \geq 0\right\} \text {. }
$$

Note that the vectors $\hat{e}_{S_{\mathcal{O}}}$ and $-\hat{\xi}$ are not parallel if $\beta \in\left(0, \beta_{*}\right]$, thus, $\operatorname{Cone}\left(\hat{e}_{S_{\mathcal{O}}},-\hat{\xi}\right)$ has non-empty interior. Let $\operatorname{Cone}^{0}\left(\hat{e}_{S_{\mathcal{O}}},-\hat{\xi}\right)$ be the interior of Cone $\left(\hat{e}_{S_{\mathcal{O}}},-\hat{\xi}\right)$. 
Remark 4.2. For $\beta=0$, we have $\hat{e}_{S_{\mathcal{O}}}=\hat{\xi}$. Then, for $\beta=0$, we define Cone $\left(\hat{e}_{S_{\mathcal{O}}},-\hat{\xi}\right)$ by the upper half-plane, that is,

$$
\text { Cone }\left(\hat{e}_{S_{\mathcal{O}}},-\hat{\xi}\right):=\left\{\boldsymbol{u} \in \mathbb{R}^{2}: \boldsymbol{u} \cdot \hat{\eta} \geq 0\right\},
$$

and let $\operatorname{Cone}^{0}\left(\hat{e}_{S_{\mathcal{O}}},-\hat{\xi}\right)$ be the interior of the upper-half plane $\left\{\boldsymbol{u} \in \mathbb{R}^{2}: \boldsymbol{u} \cdot \hat{\eta}>\right.$ $0\}$. Note that this is consistent with the definition for $\beta>0$ in the sense that $\left(\text { Cone }^{0}\left(\hat{e}_{S_{\mathcal{O}}},-\hat{\xi}\right)\right)_{\mid \beta} \rightarrow\left(\operatorname{Cone}^{0}\left(\hat{e}_{S_{\mathcal{O}}}(\beta=0),-\hat{\xi}\right)\right)_{\mid \beta=0}$ as $\beta \rightarrow 0+$, where the convergence is locally in the Hausdorff metric.

Hereafter, we assume that $\varphi$ is a weak solution satisfying properties (i)-(v) in Theorem 1, unless otherwise specified. By Lemma 4.1, we have

$$
\begin{array}{ll}
\partial_{e}\left(\varphi_{\infty}-\varphi\right)<0 & \text { in } \bar{\Omega} \text { for all } e \in \operatorname{Cone}^{0}\left(\hat{e}_{S_{\mathcal{O}}},-\hat{\xi}\right), \\
\partial_{\boldsymbol{e}}\left(\varphi-\varphi_{\mathcal{N}}\right) \geq 0 & \text { in } \bar{\Omega} \text { for all } e \in \operatorname{Cone}(-\hat{\xi},-\hat{\eta}), \\
\partial_{\boldsymbol{e}}\left(\varphi-\varphi_{\mathcal{O}}\right) \geq 0 & \text { in } \bar{\Omega} \text { for all } e \in \operatorname{Cone}\left(\hat{e}_{S_{\mathcal{O}}},-\hat{\eta}\right) .
\end{array}
$$

By (2.16) and the first inequality in (4.1), if $\varphi$ is a weak solution with properties (i)-(v) in Theorem 1 for $\left(v_{\infty}, \beta\right) \in \mathfrak{P}_{2}$, there exists a function $\eta=f_{\text {shock }}(\xi)$ satisfying

(i) $\Gamma_{\text {shock }}=\left\{(\xi, \eta): \xi \in\left(\xi_{P_{1}}, \xi_{P_{2}}\right), \eta=f_{\text {shock }}(\xi)\right\}$, where $\xi_{P_{j}}$ is the $\xi$-coordinate of the point $P_{j}$ for $j=1,2$;

(ii) $f_{\text {shock }}(\xi)$ is strictly monotone in $\xi \in\left(\xi_{\mathcal{O}}, \xi_{\mathcal{N}}\right)$ :

$$
f_{\text {shock }}^{\prime}(\xi)>0 \quad \text { for all } \xi \in\left(\xi_{\mathcal{O}}, \xi_{\mathcal{N}}\right) .
$$

(iii) there exists a constant $C_{1}$ depending only on $\gamma, v_{\infty}$, and $\beta_{*}$ such that

$$
\sup _{\xi_{\mathcal{O}}<\xi<\xi_{\mathcal{N}}}\left|f_{\text {shock }}^{\prime}(\xi)\right| \leq C_{1}
$$

From (4.2), it easily follows that

$$
\inf _{\beta \in\left[0, \beta_{*}\right]} \operatorname{dist}\left(\Gamma_{\text {shock }}, \Gamma_{\text {wedge }}\right) \geq \inf _{\left[0, \beta_{*}\right]} \eta_{P_{1}}>0 .
$$

Moreover, (4.2) implies that the region $\Omega$ enclosed by $\Gamma_{\text {shock }}, \Gamma_{\text {sonic }}^{\mathcal{O}}, \Gamma_{\text {sonic }}^{\mathcal{N}}$, and $\Gamma_{\text {wedge }}$ is uniformly bounded for all $\beta \in\left(0, \beta_{*}\right]$. In fact, we have

$$
\Omega \subset\left\{(\xi, \eta): u_{\mathcal{O}}-c_{\mathcal{O}}<\xi<c_{\mathcal{N}}, 0<\eta<\eta_{\mathcal{N}}\right\} .
$$

From this, we obtain a constant $C>0$ depending only on $\gamma, v_{\infty}$, and $\beta_{*}$ such that, if $\varphi$ is a weak solution with properties (i)-(v) in Theorem 1 for $\beta \in\left(0, \beta_{*}\right]$, then

$$
\begin{aligned}
& \Omega \subset B_{C}(\mathbf{0}), \\
& \sup _{\Omega}|\varphi| \leq C, \quad\|\varphi\|_{C^{0,1}(\bar{\Omega})} \leq C \\
& \left(\frac{2}{\gamma+1}\right)^{\frac{1}{\gamma-1}} \leq \rho \leq C \text { in } \Omega, \quad 1 \leq \rho \leq C \text { on } \Gamma_{\text {shock }} .
\end{aligned}
$$
4.3.

The importance of the last two inequalities in (4.1) will be mentioned in Section 
4.2. Uniform lower bound for the distance between the shock and the sonic circle of the upstream state. We can establish the following crucial proposition.

Proposition 4.3. Given $\gamma \geq 1$ and $v_{\infty}>0$, if $\varphi$ is a weak solution for $\beta \in\left(0, \beta_{*}\right]$ with properties (i)-(v) in Theorem 1, then

$$
\operatorname{dist}\left(\Gamma_{\text {shock }}, B_{1}\left(0,-v_{\infty}\right)\right) \geq \frac{1}{C}>0 .
$$

Proposition 4.3 is essential to obtain uniform ellipticity constants of equation (2.8). More precisely, we first write equation (2.8) with $\rho$ given by (2.13) and $\rho_{\infty}=1$ as

$$
\operatorname{div} \mathcal{A}(D \varphi, \varphi, \xi, \eta)+\mathcal{B}(D \varphi, \varphi, \xi, \eta)=0
$$

for $\mathbf{p}=\left(p_{1}, p_{2}\right) \in \mathbb{R}^{2}, z \in \mathbb{R}$, and $(\xi, \eta) \in \mathbb{R}^{2}$, where

$$
\mathcal{A}(\mathbf{p}, z, \xi, \eta) \equiv \mathcal{A}(\mathbf{p}, z):=\rho\left(|\mathbf{p}|^{2}, z\right) \mathbf{p}, \quad \mathcal{B}(\mathbf{p}, z, \xi, \eta) \equiv \mathcal{B}(\mathbf{p}, z):=2 \rho\left(|\mathbf{p}|^{2}, z\right) .
$$

Then, using (4.5), we can find a constant $C$ such that, if $\varphi$ is a weak solution for $\beta \in\left(0, \beta_{*}\right]$ and a set $U \subset \bar{\Omega}$ satisfies $\operatorname{dist}\left(U, \Gamma_{\text {sonic }}^{\mathcal{O}} \cup \Gamma_{\text {sonic }}^{\mathcal{N}}\right) \geq d>0$, we have

$$
\frac{d}{C}|\kappa|^{2} \leq \sum_{i, j=1}^{2} \mathcal{A}_{p_{j}}^{i}(D \varphi(\xi, \eta), \varphi(\xi, \eta)) \kappa_{i} \kappa_{j} \leq C|\kappa|^{2}
$$

for $(\xi, \eta) \in U$ and any $\boldsymbol{\kappa}=\left(\kappa_{1}, \kappa_{2}\right) \in \mathbb{R}^{2}$. The important part is that such a constant $C$ can be chosen depending only on $\gamma, v_{\infty}$, and $\beta_{*}$, but independent of $\varphi$ and $d$; therefore we obtain the uniform estimates of weak solutions of Problem 3 for $\beta \in\left(0, \beta_{*}\right]$.

4.3. Uniform Estimates of Global Weak Solutions. We fix $v_{\infty}>0, \gamma \geq 1$, and $\beta_{*} \in\left(0, \beta_{\text {sonic }}\left(v_{\infty}\right)\right)$. Thanks to (4.8), we can achieve uniform estimates of weak solutions $\varphi$ in $\Omega$ for $\beta \in\left(0, \beta_{*}\right]$. Because of the degeneracy of the ellipticity of equation (2.8) on $\Gamma_{\text {sonic }}^{\mathcal{O}} \cup \Gamma_{\text {sonic }}^{\mathcal{N}}$, we need to consider two cases:

Case 1: Away from $\Gamma_{\text {sonic }}^{\mathcal{O}} \cup \Gamma_{\text {sonic }}^{\mathcal{N}}$, where equation (2.8) is uniformly elliptic;

Case 2: Near $\Gamma_{\text {sonic }}^{\mathcal{O}} \cup \Gamma_{\text {sonic }}^{\mathcal{N}}$, where the ellipticity degenerates.

4.3.1. $C^{2, \alpha}$-estimates away from $\Gamma_{\text {sonic }}^{\mathcal{O}} \cup \Gamma_{\text {sonic }}^{\mathcal{N}}$. By (4.8), equation (2.8) is uniformly elliptic away from the sonic $\operatorname{arcs} \Gamma_{\text {sonic }}^{\mathcal{O}} \cup \Gamma_{\text {sonic }}^{\mathcal{N}}$. Then we can show that, for any $\alpha \in(0,1)$ and $r>0$, there exists a constant $C>0$ depending only on $\gamma, v_{\infty}, \beta_{*}, \alpha$, and $r$ such that, if $\varphi$ is a weak solution with properties (i)-(v) in Theorem 1 for $\beta \in\left(0, \beta_{*}\right]$, then we have

(i) for any $B_{2 r}(P) \subset \Omega$,

$$
\|\varphi\|_{C^{2, \alpha}\left(\overline{B_{r}(P)}\right)} \leq C
$$

(ii) for $P \in \Gamma_{\text {wedge }}$ and $\left(B_{2 r}(P) \cap \Omega\right) \cap\left(\Gamma_{\text {sonic }}^{\mathcal{O}} \cup \Gamma_{\text {sonic }}^{\mathcal{N}}\right)=\emptyset$,

$$
\|\varphi\|_{C^{2, \alpha}\left(\overline{B_{r}(P) \cap \Omega}\right)} \leq C .
$$


For uniform estimates of shock curves $\Gamma_{\text {shock }}$ and weak solutions $\varphi$ on $\Gamma_{\text {shock}}$, we use a partial hodograph transform. For any $\beta \in\left(0, \beta_{\text {sonic }}\right)$, we have

$$
\hat{\eta} \in \operatorname{Cone}^{0}\left(\hat{e}_{S_{\mathcal{O}}},-\hat{\xi}\right) .
$$

Then, by the first inequality in (4.1), we can find constants $\delta$ and $\sigma>0$ depending only on $\gamma, v_{\infty}$, and $\beta_{*}$ such that, if $\varphi$ is a weak solution for $\beta \in\left(0, \beta_{*}\right]$, then

$$
\partial_{\eta}\left(\varphi_{\infty}-\varphi\right) \leq-\delta \quad \text { in } \mathcal{N}_{\sigma}\left(\Gamma_{\text {shock }}\right) \cap \Omega
$$

for $\mathcal{N}_{\sigma}\left(\Gamma_{\text {shock }}\right)=\left\{(\xi, \eta) \in \mathbb{R}^{2}: \operatorname{dist}\left((\xi, \eta), \Gamma_{\text {shock }}\right)<\sigma\right\}$. We introduce a coordinate system $\left(\xi^{\prime}, \eta^{\prime}\right)$ with $\xi^{\prime}=\xi$ and a function $v\left(\xi^{\prime}, \eta^{\prime}\right)$ such that $v$ satisfies

$$
v\left(\xi,\left(\varphi_{\infty}-\varphi\right)(\xi, \eta)\right)=\eta
$$

By (4.9), such a coordinate system $\left(\xi^{\prime}, \eta^{\prime}\right)$ is well defined. In the new coordinates, we use equation (2.8) in the set $\mathcal{N}_{\sigma}\left(\Gamma_{\text {shock }}\right) \cap \Omega$ and the Rankine-Hugoniot condition:

$$
\rho D \varphi \cdot \nu_{s}=D \varphi_{\infty} \cdot \boldsymbol{\nu}_{s} \quad \text { on } \Gamma_{\text {shock }}
$$

to obtain an equation and a boundary condition for $v$. Then we obtain an elliptic equation and an oblique boundary condition for $v$ in the $\left(\xi^{\prime}, \eta^{\prime}\right)$-coordinates. By (2.16) $), \Gamma_{\text {shock }}$ becomes a fixed flat boundary; then we obtain the uniform estimates of $v$ for $\beta \in\left[0, \beta_{*}\right]$. By (4.10), we obtain $\Gamma_{\text {shock }}=\{\eta=v(\xi, 0)\}$; thus the uniform estimates of $v$ imply that, for any $k \in \mathbb{N}$ and $d>0$, there are constants $s, C_{k}(d)$, and $\hat{C}_{k}(d)$ depending on $\gamma, v_{\infty}, \beta_{*}$, and $d$ such that, if $P\left(\xi_{P}, \eta_{P}\right) \in \Gamma_{\text {shock }}$ and $\operatorname{dist}\left(P, \Gamma_{\text {sonic }}^{\mathcal{O}} \cup \Gamma_{\text {sonic }}^{\mathcal{N}}\right) \geq d$, then

$$
\left|D^{k} f_{\text {shock }}\left(\xi_{P}\right)\right| \leq C_{k}(d), \quad\left|D_{(\xi, \eta)}^{k} \varphi\right| \leq \hat{C}_{k}(d) \quad \text { on } B_{s}(P) \cap \Omega,
$$

where $C_{k}(d)$ and $\hat{C}_{k}(d)$ depend additionally on $k$.

4.3.2. Weighted $C^{2, \alpha}$-estimates near $\Gamma_{\text {sonic }}^{\mathcal{O}} \cup \Gamma_{\text {sonic. }}^{\mathcal{N}}$ Near the sonic $\operatorname{arcs} \Gamma_{\text {sonic }}^{\mathcal{O}} \cup$ $\Gamma_{\text {sonic }}^{\mathcal{N}}$, it is convenient to use the coordinates in which the sonic arcs are flattened. For that reason, we introduce the $(x, y)$-coordinates as follows: Choose $\varepsilon>0$ small.

- For $(\xi, \eta) \in \mathcal{N}_{\varepsilon}\left(\Gamma_{\text {sonic }}^{\mathcal{N}}\right):=\left\{(\xi, \eta): \operatorname{dist}\left((\xi, \eta), \Gamma_{\text {sonic }}^{\mathcal{N}}\right)<\varepsilon\right\}$, we set

$$
x:=c_{\mathcal{N}}-r, \quad y:=\theta
$$

for the polar coordinates $(r, \theta)$ centered at $(0,0)$;

- For $(\xi, \eta) \in \mathcal{N}_{\varepsilon}\left(\Gamma_{\text {sonic }}^{\mathcal{O}}\right):=\left\{(\xi, \eta): \operatorname{dist}\left((\xi, \eta), \Gamma_{\text {sonic }}^{\mathcal{O}}\right)<\varepsilon\right\}$, we set

$$
x:=c_{\mathcal{O}}-r, \quad y:=\pi-\theta
$$

for the polar coordinates $(r, \theta)$ centered at $\left(u_{\mathcal{O}}, 0\right)$.

From the definition of $(x, y)$ above, it is easy to see that

$$
\begin{aligned}
& \left(\Omega \cap \mathcal{N}_{\varepsilon}(\Gamma)\right) \subset\{x>0, y>0\}, \quad \Gamma=\bar{\Omega} \cap\{x=0\}, \\
& \left(\{\eta=0\} \cap \mathcal{N}_{\varepsilon}(\Gamma)\right)=\bar{\Omega} \cap\{y=0\}
\end{aligned}
$$

for $\Gamma=\Gamma_{\text {sonic }}^{\mathcal{O}}$ or $\Gamma_{\text {sonic }}^{\mathcal{N}}$. For $\varphi_{\mathcal{O}}$ and $\varphi_{\mathcal{N}}$ defined by (2.20) and (2.23), respectively, set

$$
\psi:=\varphi-\max \left(\varphi_{\mathcal{O}}, \varphi_{\mathcal{N}}\right)
$$


In fact, choosing $\varepsilon$ sufficiently small, we have

$$
\max \left(\varphi_{\mathcal{O}}, \varphi_{\mathcal{N}}\right)= \begin{cases}\varphi_{\mathcal{O}} & \text { in } \mathcal{N}_{\varepsilon}\left(\Gamma_{\text {sonic }}^{\mathcal{O}}\right) \cap \Omega, \\ \varphi_{\mathcal{N}} & \text { in } \mathcal{N}_{\varepsilon}\left(\Gamma_{\text {sonic }}^{\mathcal{N}}\right) \cap \Omega .\end{cases}
$$

This implies that

$$
\psi=\varphi-\varphi_{\mathcal{O}} \text { in } \mathcal{N}_{\varepsilon}\left(\Gamma_{\text {sonic }}^{\mathcal{O}}\right) \cap \Omega, \quad \psi=\varphi-\varphi_{\mathcal{N}} \text { in } \mathcal{N}_{\varepsilon}\left(\Gamma_{\text {sonic }}^{\mathcal{N}}\right) \cap \Omega .
$$

Since we seek a weak solution $\varphi$ of Problem 3 to be $C^{1}$ across $\Gamma_{\text {sonic }}^{\mathcal{N}} \cup \Gamma_{\text {sonic }}^{\mathcal{O}}, \psi$ satisfies

$$
\psi(0, y) \equiv 0 .
$$

Using the definition of $\psi$, we can rewrite equation (2.8) as an equation for $\psi$ in the $(x, y)$-coordinates near $\Gamma_{\text {sonic }}^{\mathcal{O}}$ or $\Gamma_{\text {sonic }}^{\mathcal{N}}$ as follows:

$$
A_{11}(D \psi, \psi, x) \psi_{x x}+2 A_{12}(D \psi, \psi, x) \psi_{x y}+A_{22}(D \psi, \psi, x) \psi_{y y}+A(D \psi, \psi, x)=0,
$$

where $\left(A_{i j}, A\right)(\boldsymbol{p}, z, x)$ are smooth with respect to $(\boldsymbol{p}, z, x)$. Then (4.8) implies

$$
\lambda|\boldsymbol{\kappa}|^{2} \leq \frac{A_{11}(D \psi, \psi, x)}{x} \kappa_{1}^{2}+2 \frac{A_{12}(D \psi, \psi, x)}{\sqrt{x}} \kappa_{1} \kappa_{2}+A_{22}(D \psi, \psi, x) \kappa_{2}^{2} \leq \frac{1}{\lambda}|\boldsymbol{\kappa}|^{2}
$$

for a constant $\lambda>0$, where $\lambda$ depends only on $v_{\infty}, \gamma$, and $\beta_{*}$. Using the expressions of the coefficients $A_{i j}(\boldsymbol{p}, z, x)$ and the fact that an ellipticity constant in (4.8) is proportional to the distance to the sonic $\operatorname{arcs} \Gamma_{\text {sonic }}^{\mathcal{O}} \cup \Gamma_{\text {sonic }}^{\mathcal{N}}$, we obtain a constant $\delta>0$ such that, for any $\beta \in\left(0, \beta_{*}\right]$, a corresponding $\psi$ satisfies

$$
\psi_{x} \leq \frac{2-\delta}{1+\gamma} x \quad \text { in } \mathcal{N}_{\varepsilon}\left(\Gamma_{\text {sonic }}^{\mathcal{O}} \cup \Gamma_{\text {sonic }}^{\mathcal{N}}\right) \cap \Omega,
$$

and such $\delta>0$ depends only on $\gamma, v_{\infty}$, and $\beta_{*}$. Also, by the last two inequalities in (4.1) and the definition of the $(x, y)$-coordinates, one can easily check that $\psi_{x} \geq 0$ in $\mathcal{N}_{\varepsilon}\left(\Gamma_{\text {sonic }}^{\mathcal{O}} \cup \Gamma_{\text {sonic }}^{\mathcal{N}}\right) \cap \Omega$. Combining this with (4.15), we obtain

$$
\left|\psi_{x}\right| \leq C x \quad \text { in } \Omega \cap \mathcal{N}_{\varepsilon}\left(\Gamma_{\text {sonic }}^{\mathcal{N}} \cup \Gamma_{\text {sonic }}^{\mathcal{O}}\right) \text {. }
$$

Combining (4.16) with (4.13), we obtain

$$
|\psi(x, y)| \leq C x^{2} \quad \text { in } \mathcal{N}_{\varepsilon}\left(\Gamma_{\text {sonic }}^{\mathcal{O}} \cup \Gamma_{\text {sonic }}^{\mathcal{N}}\right) \cap \Omega .
$$

It is important to note that the constant $C$ in (4.16) and (4.17) depend only on $\gamma, v_{\infty}$, and $\beta_{*}$. Then the uniform weighted $C^{2, \alpha}$-estimates of $\psi$ for $\beta \in\left(0, \beta_{*}\right]$ are obtained by the local scaling of $\psi$ and the covering argument.

For $P_{0} \in \mathcal{N}_{\varepsilon}\left(\Gamma_{\text {sonic }}^{\mathcal{O}} \cup \Gamma_{\text {sonic }}^{\mathcal{N}}\right) \cap\left(\bar{\Omega} \backslash\left(\Gamma_{\text {sonic }}^{\mathcal{O}} \cup \Gamma_{\text {sonic }}^{\mathcal{N}}\right)\right)$, set $P_{0}:=\left(x_{0}, y_{0}\right)$ in the $(x, y)$-coordinates and define

$$
\psi^{\left(x_{0}, y_{0}\right)}(S, T)=\frac{1}{d^{2}} \psi\left(x_{0}+d S, y_{0}+\sqrt{d} T\right) \quad \text { with } d=\frac{x_{0}}{10}
$$

in $Q_{1}^{\left(x_{0}, y_{0}\right)}:=\left\{(S, T) \in(-1,1)^{2}:\left(x_{0}+d S, y_{0}+\sqrt{d} T\right) \in \Omega\right\}$. For the estimates of

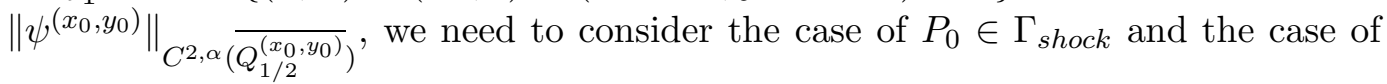
$P_{0} \notin \Gamma_{\text {shock}}$, separately. For $P_{0} \in \Gamma_{\text {shock}}$, we use the first inequality in (4.1) to show that

$$
\partial_{y}\left(\varphi_{\infty}-\varphi\right) \geq \delta \quad \text { near } \Gamma_{\text {sonic }}^{\mathcal{O}} \text { or } \Gamma_{\text {sonic }}^{\mathcal{N}} \text { for some } \delta>0 .
$$


Then, as in (4.10), we can introduce a coordinate system $\left(x^{\prime}, y^{\prime}\right)$ and a function $w\left(x^{\prime}, y^{\prime}\right)$ satisfying

$$
w\left(x,\left(\varphi_{\infty}-\varphi\right)(x, y)\right)=y,
$$

and we write an equation and a boundary condition for $w$ by using equation (2.8) and the boundary condition (4.11). And, we take the same procedure to the function $\varphi_{\infty}-\max \left(\varphi_{\mathcal{O}}, \varphi_{\mathcal{N}}\right)$ to introduce a function $w_{0}$ and its equation. Then we take the difference of the equations for $w$ and $w_{0}$ to obtain an equation for $w-w_{0}$ and use it to estimate $w-w_{0}$. This procedure will provide the weighted $C^{2, \alpha}$-estimates of $\Gamma_{\text {shock }}$ near $P_{0}$ and $\left\|\psi^{\left(x_{0}, y_{0}\right)}\right\|_{C^{2, \alpha}\left(\overline{\left.Q_{1 / 2}^{\left(x_{0}, y_{0}\right)}\right)}\right.}$.

For $P_{0} \in \Omega \cup \Gamma_{\text {wedge }}$, it is easier to obtain the estimates of $\left\|\psi^{\left(x_{0}, y_{0}\right)}\right\|_{C^{2, \alpha}\left(\overline{\left.Q_{1 / 2}^{\left(x_{0}, y_{0}\right)}\right)}\right.}$. Then, the supremum of $\left\|\psi^{\left(x_{0}, y_{0}\right)}\right\|_{C^{2, \alpha}\left(\overline{Q^{\left(x_{0}, y_{0}\right)}}\right)}$ over $\left(x_{0}, y_{0}\right) \in \mathcal{N}_{\varepsilon}\left(\Gamma_{\text {sonic }}^{\mathcal{O}} \cup \Gamma_{\text {sonic }}^{\mathcal{N}}\right) \cap \Omega$ is uniformly bounded for any $\beta \in\left(0, \beta_{*}\right]$. We define

$$
\|\psi\|_{2, \alpha, \mathcal{N}_{\varepsilon}(\Gamma) \cap \Omega}^{(\text {par })}:=\sup _{\left(x_{0}, y_{0}\right) \in \mathcal{N}_{\varepsilon}(\Gamma) \cap \Omega}\left\|\psi^{\left(x_{0}, y_{0}\right)}\right\|_{C^{2, \alpha}\left(\overline{Q_{1 / 2}^{\left(x_{0}, y_{0}\right)}}\right)}
$$

for $\Gamma=\Gamma_{\text {sonic }}^{\mathcal{O}} \cup \Gamma_{\text {sonic }}^{\mathcal{N}}$. We note that the uniform estimate of $\|\psi\|_{2, \alpha, \mathcal{N}_{\varepsilon}(\Gamma) \cap \Omega}^{(\text {par })}$ automatically provides the uniform $C^{1,1}$-estimates of $\psi$ in $\mathcal{N}_{\varepsilon}(\Gamma) \cap \Omega$ for $\beta \in\left(0, \beta_{*}\right]$.

4.4. Outline of the proof of Theorem 1. To establish Theorem 1, that is, the existence of a weak solution of Problem 3 for all $\left(v_{\infty}, \beta\right) \in \mathfrak{P}_{2}$, we apply an iteration procedure. For that purpose, we define an iteration set $\mathcal{K}$ and an iteration map $\mathcal{F}$ on $\mathcal{K}$ so that a fixed point of $\mathcal{F}$ yields a solution of Problem 3 , Then, we apply the Leray-Schauder degree theorem to show the existence of fixed points.

Given $\gamma \geq 1$ and $\left(v_{\infty}, \beta\right) \in \mathfrak{P}_{2}$, let $\varphi$ be a solution of Problem 3 with a shock $\Gamma_{\text {shock }}$ satisfying (i)-(v) of Theorem 1, By (ii) of Problem 3, $\Gamma_{\text {shock }}$ is determined by the solution $\varphi$. Moreover, by the monotonicity property (4.1), $\Gamma_{\text {shock }}$ is a graph in $\eta$-direction, so we can introduce an invertible mapping $\mathfrak{G}$ which maps $\Omega$ onto the rectangular domain $\mathcal{Q}^{i t e r}:=(-1,1) \times(0,1)$. Then the function $U=\varphi \circ \mathfrak{G}^{-1}$ is well defined on $\mathcal{Q}^{\text {iter }}$ regardless of $\beta$. In other words, any solution of Problem 3 satisfying (i)-(v) of Theorem 1 can be re-defined as a function on $\mathcal{Q}^{\text {iter }}$. This allows us to perform iteration in a set of functions defined in $\mathcal{Q}^{i t e r}$ to prove the existence of a solution of Problem 3 ,

We define the iteration set $\mathcal{K}$ as a product of a parameter set and a set of functions defined in $\mathcal{Q}^{\text {iter }}$. Given $\gamma \geq 1$ and $v_{\infty}>0$, we fix $\beta_{*} \in\left(0, \beta_{\text {sonic }}\left(v_{\infty}\right)\right)$ and set

$$
\mathcal{K}:=\cup_{\beta \in\left[0, \beta_{*}\right]}\{\beta\} \times \mathcal{K}(\beta),
$$

where $\mathcal{K}(\beta)$ is a set of functions defined in $\mathcal{Q}^{\text {iter }}$. We define $\mathcal{K}(\beta)$ such that, if $u \in \mathcal{K}(\beta)$, there exist a corresponding pseudo-subsonic region $\Omega^{(u)}$ and a function $\varphi^{(u)}$ defined in $\Omega^{(u)}$, which satisfies several properties including the monotonicity properties (4.1), so that it can be a candidate of solution of Problem 3 satisfying (i) - (v) of Theorem 1 .

Once the iteration set $\mathcal{K}$ is defined, the iteration map $\mathcal{F}$ is defined as follows: Given $(\beta, u) \in \mathcal{K}$, define the corresponding pseudo-subsonic domain $\Omega^{(u)}$, enclosed 
by $\Gamma_{\text {sonic }}^{\mathcal{N}}, \Gamma_{\text {sonic }}^{\mathcal{O}}$, and $\Gamma_{\text {wedge }}$, and an approximate shock $\Gamma_{\text {shock }}^{(u)}$, and set up a boundary value problem for an elliptic equation whose ellipticity degenerates on $\Gamma_{\text {sonic }}^{\mathcal{N}} \cup$ $\Gamma_{\text {sonic }}^{\mathcal{O}}$. Let $\tilde{\varphi}$ be the solution of the boundary value problem in $\Omega^{(u)}$. The iteration set $\mathcal{K}$ will be defined so that such $\tilde{\varphi}$ exists. Then we can define a function $\tilde{u}$ in $\mathcal{Q}^{i t e r}$ from $\tilde{\varphi}$. Then the iteration map $\mathcal{F}$ is defined by $\mathcal{F}(\beta, u)=\tilde{u}$. The boundary value problem for $\tilde{\varphi}$ is set up so that, if $u=\tilde{u}$, then $\tilde{\varphi}$ satisfies equation (2.8), with an ellipticity cutoff in a small neighborhood of $\Gamma_{\text {sonic }}^{\mathcal{O}} \cup \Gamma_{\text {sonic }}^{\mathcal{N}}$, the Rankine-Hugoniot conditions (2.15) -(2.16) on the shock $\Gamma_{\text {shock }}$, and the boundary conditions stated in (iii)-(iv) of Problem 3, More specifically, note that two conditions in (iii) of Problem 3 are specified on the sonic arcs. Since the sonic arcs are fixed boundaries, this looks like an overdeterminacy. Thus, for the iteration problem, we use only the Dirichlet condition

$$
\tilde{\varphi}=\varphi_{\beta}=\max \left(\varphi_{\mathcal{O}}, \varphi_{\mathcal{N}}\right) \quad \text { on } \Gamma_{\text {sonic }}^{\mathcal{O}} \cup \Gamma_{\text {sonic }}^{\mathcal{N}}
$$

and then we prove that the condition $D \tilde{\varphi}=D \varphi_{\beta}$ on $\Gamma_{\text {sonic }}^{\mathcal{O}} \cup \Gamma_{\text {sonic }}^{\mathcal{N}}$ also holds. In this proof, we use the elliptic degeneracy of the equation in $\Omega^{(u)}$ near $\Gamma_{\text {sonic }}^{\mathcal{O}} \cup \Gamma_{\text {sonic }}^{\mathcal{N}}$. Technically, this follows from the estimates of $\tilde{\psi}=\tilde{\varphi}-\varphi_{\beta}$ in norms (4.18) near $\Gamma_{\text {sonic }}^{\mathcal{O}} \cup \Gamma_{\text {sonic }}^{\mathcal{N}}$.

If $\left(\beta, u_{*}\right) \in \mathcal{K}$ satisfies $\mathcal{F}\left(\beta, u_{*}\right)=u_{*}$, then we call $u_{*}$ a fixed point. In order for a fixed point $u_{*}$ to provide a solution of Problem 3, we need to show that $\varphi_{*}$ satisfies equation (2.8) without the ellipticity cutoff. Moreover, since we intend to apply the Leray-Schauder degree theorem to establish Theorem 1, we need to prove the following properties:

(i) If $u_{*} \in \mathcal{K}(\beta)$ is a fixed point of $\mathcal{F}$ for some $\beta \in\left[0, \beta_{*}\right]$, then the corresponding $\varphi_{*}$ satisfies equation (2.8) without the ellipticity cutoff;

(ii) For any $\beta \in\left[0, \beta_{*}\right]$, the map $\mathcal{F}(\beta, \cdot)$ is compact, and $\mathcal{F}$ is continuous;

(iii) The iteration set $\mathcal{K}$ is open;

(iv) For any $\beta \in\left[0, \beta_{*}\right]$, there is no fixed point of $\mathcal{F}$ on the boundary of the iteration set $\mathcal{K}(\beta)$.

In proving all the properties above, the apriori estimates in Sections 4.1 and 4.2 play an essential role. Once (i)-(iv) are verified, then $\operatorname{deg}(\mathcal{F}(\beta, \cdot)-I d, \mathcal{K}(\beta), 0)$ is a constant for all $\beta \in\left[0, \beta_{*}\right]$.

To complete the proof of Theorem 1 , we show that $\operatorname{deg}(\mathcal{F}(0, \cdot)-I d, \mathcal{K}(0), 0) \neq 0$ in two steps. First, we prove that $\mathcal{F}(0, \cdot)$ has the unique fixed point $u^{(\text {normal })}$ in $\mathcal{K}(0)$. In fact, we have $u^{(\text {normal })} \equiv 0$. Then we can conclude that $\operatorname{deg}(\mathcal{F}(0, \cdot)-I d, \mathcal{K}(0), 0) \neq 0$ by showing that $D_{u} \mathcal{F}\left(0, u^{(\text {normal })}\right)-I$ has the trivial kernel.

As mentioned earlier, once we establish Theorem 1, that is, the existence of global weak solutions of Problem 3, we conclude the existence of a global self-similar weak shock solution for any admissible wedge angle $\theta_{w}$ by the one-to-one correspondence between the parameter sets $\mathfrak{P}_{1}$ and $\mathfrak{P}_{2}$.

For the detailed proof, see Bae-Chen-Feldman [2].

Acknowledgments. The research of Gui-Qiang G. Chen was supported in part by the National Science Foundation under Grants DMS-0935967 and DMS0807551, the UK EPSRC Science and Innovation Award to the Oxford Centre for 
Nonlinear PDE (EP/E035027/1), the NSFC under a joint project Grant 10728101, and the Royal Society-Wolfson Research Merit Award (UK). The work of Mikhail Feldman was supported in part by the National Science Foundation under Grants DMS-0800245, DMS-1101260, and the Vilas Award by the University of WisconsinMadison. The research of Myoungjean Bae was supported in part by the initial settlement research fund provided by POSTECH.

\section{REFERENCES}

[1] Bae, M., Chen, G.-Q. and Feldman, M., Regularity of solutions to regular shock reflection for potential flow, Invent. Math. 175 (2009), 505-543.

[2] Bae, M., Chen, G.-Q. and Feldman, M., Global solutions to the Prandtl-Meyer reflection for supersonic potential flow impinging onto a solid wedge, In preparation, 2012.

[3] Busemann, A. Gasdynamik, Handbuch der Experimentalphysik, Vol. IV, Akademische Verlagsgesellschaft, Leipzig, 1931.

[4] Chen, G.-Q. and Feldman, M., Potential theory for shock reflection by a large-angle wedge, Proc. Nat. Acad. Sci. U.S.A. 102 (2005), 15368-15372.

[5] Chen, G.-Q. and Feldman, M., Global solutions to shock reflection by large-angle wedges for potential flow, Ann. Math. 171 (2010), 1019-1134.

[6] Chen, G.-Q. and Feldman, M., Mathematics of Shock Reflection-Diffraction, von Neumann's Conjectures, and Related Analysis, Monograph, Oxford 2011.

[7] Chen, G.-Q. and Li, T.-H., Well-posedness for two-dimensional steady supersonic Euler flows past a Lipschitz wedge, J. Diff. Eqs. 244 (2008), 1521-1550.

[8] Chen, G.-Q., Zhang, Y., and Zhu, D., Existence and stability of supersonic Euler flows past Lipschitz wedges, Arch. Ration. Mech. Anal. 181 (2006), 261-310.

[9] Chen, S. and Fang, B., Stability of transonic shocks in supersonic flow past a wedge, J. Diff. Eqs. 233 (2007), 105C135.

[10] Courant, R. and Friedrichs, K. O., Supersonic Flow and Shock Waves, SpringerVerlag: New York, 1948.

[11] Dafermos, C. M., Hyperbolic Conservation Laws in Continuum Physics, Third edition, Springer-Verlag: Berlin, 2010.

[12] Elling, V. and Liu, T.-P., The ellipticity principle for self-similar potential flows, J. hyperbolic Differ. Equ. 2 (2005), 909-917.

[13] Elling, V and Liu, T.-P., Supersonic flow onto a solid wedge, Comm. Pure Appl. Math. 61 (2008), 1347-1448.

[14] Lieberman, G. M., Hölder continuity of the gradient at a corner for the capillary problem and related result, Pacific J. Math. 133 (1988), 115-135.

[15] Meyer, Th., Über zweidimensionale Bewegungsvorgänge in einem Gas, das mit Überschallgeschwindigkeit strömt. Dissertation, Göttingen, 1908. Forschungsheft des Vereins deutscher Ingenieure, Vol. 62, Berlin, 1908, pp. 31-67.

[16] Prandtl, L., Allgemeine Überlegungen über die Strömung zusammendrückbarer Flüssigkeiten, Zeitschrift für angewandte Mathematik und Mechanik, 16 (1936), 129142.

[17] Serre, D., Von Neumann's comments about existence and uniqueness for the initialboundary value problem in gas dynamics, Bull. Amer. Math. Soc. (N.S.), 47 (2010), 139-144.

[18] Whitham, G. B., Linear and Nonlinear Waves, Wiley-Interscience [John Wiley \& Sons]: New York-London-Sydney, 1974. 
M. Bae, Department of Mathematics, Postech, San 31, Hyojadong, NAmgu, PoHAng, Gyungbuk, Korea

E-mail address: mjbae@postech.ac.kr

G.-Q. Chen, Mathematical Institute, University of Oxford, 24-29 St Giles', Oxford, OX1 3LB, England; School of Mathematical Sciences, Fudan University, Shanghai 200433, China; Department of Mathematics, Northwestern University, 2033 Sheridan RoAD, Evanston, IL 60208-2734, USA

E-mail address: chengq@maths.ox.ac.uk

M. Feldman, Department of Mathematics, University of Wisconsin, Madison, Wi 53706-1388, USA

E-mail address: feldman@math.wisc.edu 